\title{
The relationship between vitamin D level and organ-specific autoimmune disorders in newly diagnosed type I diabetes mellitus
}

\author{
Akdere $\mathrm{G}^{1}$, Efe $\mathrm{B}^{2}$, Sisman $\mathrm{P}^{3}$, Yorulmaz $\mathrm{G}^{2}$ \\ Harakani State Hospital, Internal Medicine Clinic, Kars, Turkey. drpinarsisman@gmail.com
}

\begin{abstract}
INTRODUCTION: The purpose of our study is to determine vitamin D levels in patients with newly diagnosed type $1 \mathrm{DM}$, and assess the association of type $1 \mathrm{DM}$ with organ-specific autoimmune disorders, as well as their association with vitamin $D$.

MATERIAL AND METHODS: We included a total of 160 patients, of whom 50 were newly diagnosed with type 1 DM (group I), 50 were formerly diagnosed with type 1 DM (group II), and 60 were healthy controls (group III). RESULTS: The mean level of 25(OH)D was $14.6 \mathrm{ng} / \mathrm{dL}$ in group I, $12.1 \mathrm{ng} / \mathrm{dL}$ in group II, and $16.1 \mathrm{ng} / \mathrm{dL}$ in group III. In all diabetic patients, the 25(OH)D levels were lower than those of controls. The 25(OH)D median level was $11.4 \mathrm{ng} / \mathrm{ml}$ in all cases included into the study with ATD. In subjects without ATD, the latter level was $15.3 \mathrm{ng} / \mathrm{ml}$. The difference was found to be statistically significant.

CONCLUSIONS: In this study, the vitamin D level was lower in (i) type 1 diabetic patients when compared with healthy subjects, (ii) all cases included to study with ATD when compared with patients without ATD, (iii) all APA-positive type 1 diabetic patients with ATD when compared with none (iv) APA-positive newly diagnosed type 1 diabetics when compared with those APA-negative (Tab. 7, Fig. 1, Ref. 30). Text in PDF www.elis.sk. KEY WORDS: type 1 diabetes mellitus, autoimmune disease, vitamin D, anti-parietal antibody, anti-tissue transglutaminase TG-IgA.
\end{abstract}

\section{Introduction}

The presence of autoimmune disorders accompanied by type 1 diabetes mellitus (DM) has been reported. Hashimoto's thyroiditis, which most commonly affects $20-30 \%$ of patients, is reported to be detected by thyroid peroxidase antibody (anti-TPO) in $40 \%$ of patients and anti-thyroglobulin (anti-TG) autoantibody are reported positive in $33 \%$ (1). The second most common autoimmune disorder occurring in the presence of type 1 diabetes is Celiac disease. Although not common during diagnosis, studies show that celiac disease develops in $10 \%$ of patients with type $1 \mathrm{DM}$ within 5 years of diagnosis. Addison's disease (AD) is rarely accompanied by type $1 \mathrm{DM}$ and is strongly associated with HLA DR3-DQ2, the second most frequent HLA haplotype in type 1 DM (2).

The biologically active form of vitamin D, calcitriol $\left(1,25(\mathrm{OH})_{2} \mathrm{D}_{3}\right)$ has numerous physiological effects such as effects on mineral homeostasis, as well as inhibition of T lymphocyte proliferation, inflammatory cytokine production and protection against immune disease. It has also been shown to play a role in improv-

${ }^{1}$ Harakani State Hospital, Internal Medicine Clinic, Kars, Turkey, ${ }^{2} \mathrm{Os}-$ mangazi University Medical School, Department of Endocrinology and Metabolism, Eskisehir, Turkey, and ${ }^{3}$ Medicana Hospital, Endocrinology and Metabolism Clinic, Bursa, Turkey

Address for correspondence: P. Sisman, Medicana Hospital, Endocrinology and Metabolism Clinic, Bursa, Turkey.

Phone: +90.532 .1550811$ ing and maintaining the functions of immune system. However, vitamin $\mathrm{D}$ increases the beta-cell function and insulin sensitivity in target cells (liver, skeletal muscle and fat), and protects beta cells from immunization. Calcifediol $(25(\mathrm{OH}) \mathrm{D}$ vitamin) is the most sensitive test showing the vitamin D storage in the body. D vitamin deficiency is widespread at all ages, while especially low serum vitamin D levels were found in type 1 diabetic patients $(3,4)$.

While there are many studies on the relationship between type 1 diabetes and vitamin $\mathrm{D}$, there is no study of the relationship between vitamin $\mathrm{D}$ and autoimmune disorders accompanied with type $1 \mathrm{DM}$ such as autoimmune thyroid diseases (ATD), celiac disease, $\mathrm{AD}$, vitiligo and atrophic gastritis. The purpose of our study is to determine vitamin D levels in patients with newly diagnosed type $1 \mathrm{DM}$, and assess the association of type $1 \mathrm{DM}$ with organ-specific autoimmune disorders such as ATD, atrophic gastritis, AD, vitiligo and celiac disease, as well as their association with vitamin D levels.

\section{Materials and methods}

Between May 2013 and December 2015, we included a total of 160 patients, of whom 50 were newly diagnosed with type 1 $\mathrm{DM}, 50$ were formerly diagnosed with type $1 \mathrm{DM}$, and 60 were healthy controls with similar age, gender and BMI distribution. Our work has been done at our center with the approval of ethics committee with number $80558721 / 183$ and in accordance with the Declaration of Helsinki. 
Tab. 1. Distribution of the study population included in the study by groups.

\begin{tabular}{lcccc}
\hline & $\mathrm{n}(\mathrm{F} / \mathrm{M})$ & $\%$ & Age $($ year $)$ & $\mathrm{BMI}\left(\mathrm{kg} / \mathrm{m}^{2}\right)$ \\
\hline Newly diagnosed Type I DM (Group I) & $50(17 / 33)$ & 31.3 & $27(22-34.2)$ & $21.7(19.9-24)$ \\
Formerly diagnosed Type 1 DM (Group II) & $50(22 / 28)$ & 31.3 & $29(22.7-35)$ & $22(19.7-26)$ \\
Control (Group III) & $60(27 / 33)$ & 37.7 & $29(24-34.7)$ & $24(20-26.3)$ \\
\hline
\end{tabular}

The inclusion criteria of the cases are as follows: (i) adult age group (18 years and over), (ii) while there is no presence of a previously known chronic disease and drug use history, newly diagnosed type $1 \mathrm{DM}$ accompanied by the positive presence of one or more of autoantibodies such as anti-GAD, islet cell antibodies and antiinsulin antibodies, (iii) for the control group, healthy adults of age range similar to that in newly diagnosed type 1 diabetic patients, (iv) consent to participate in the study by signing the informed consent form, (v) in addition, patients with type 1 diabetes from our outpatient clinic follow-up were included retrospectively in the study by detecting the incidence of organ-specific autoimmune disorders associated with type $1 \mathrm{DM}$.

Exclusion criteria of the cases are as follows: (i) usage of drugs that can affect vitamin D levels, (ii) presence of the disease that can be associated with calcium and phosphorus metabolism, (iii) presence of kidney and liver failure, (iiii) presence of bone metabolism disorder such as rickets, osteoporosis, osteopenia or drug usage for bone metabolism disorder, (iv) primary hyperparathyroidism, (iiiiii) chronic inflammatory disease, (v) for the healthy control group, lack of known history and complaints about diabetes and thyroid disease.

The height and weight of all patients included in the study were measured by the same person using standard measuring instruments. Body mass index (BMI) was calculated using the Quetlet index (weight/height; $\mathrm{kg} / \mathrm{m}^{2}$ ). All patients and healthy subjects included in the study underwent a skin examination for hypopigmented skin areas to investigate vitiligo.

The levels of $25(\mathrm{OH}) \mathrm{D}$, calcium, phosphorus, alkaline phosphatase, parathormone (PTH), albumin, anti-TPO, free T3 (fT3), free T4 (fT4), TSH, B12 vitamin, anti-parietal antibody, anti-tissue transglutaminase IgA, and cortisol were studied after at least eight hours of fasting. Patients with an upper anti-TPO level above 34 $\mathrm{IU} / \mathrm{mL}$ were considered to be suffering from autoimmune thyroid disease. Levels of $25(\mathrm{OH}) \mathrm{D} \geq 25 \mathrm{ng} / \mathrm{ml}$ were considered adequate and those $<25 \mathrm{ng} / \mathrm{ml}$ were considered inadequate. Additional $\mathrm{HbA} 1 \mathrm{c}$ values were studied in patients with type 1 diabetes.

Blood of patients taken into a biochemical tube for the measurement of $25(\mathrm{OH}) \mathrm{D}$ vitamin was centrifuged at $3000 \mathrm{rpm}$ for 10 min and stored at $-20^{\circ} \mathrm{C}$ until analysis. At the time of the study, these serum samples were dissolved at room temperature and analyzed with ELISA method in Biotek Microplate Marked Readers (BIOTEK ELX 800 USA) with Immunodiagnostik system markers. Serum calcium, phosphorus and albumin levels were measured photometrically with Roche/Hitachi cobas c kit. HbA1c was measured with high performance liquid chromatography (HPLC) method. TSH, sT3, sT4, anti TPO, parathormone, vitamin B12, and cortisol levels were studied in electrochemiluminescence immunoassay and Elecsys and cobas e immunological test analyzers. IgA was investigated by manual ELISA method for measuring anti-tissue transglutaminase IgA. Anti-parietal antibodies were studied by indirect immunofluorescence.

\section{Statistical analysis}

All data analyses were done with IBM SPSS 21.0 package programs. Quantitative data were expressed as mean and standard deviation or as median values. The normal distribution of continuous variables was determined by the Shapiro-Wilk normality test.T test was used for comparing two groups with normal distribution and One-way ANOVA was used for comparing three groups. The Tukey test was preferred for one-way analysis of variance. MannWhitney-U was used to compare the 2 groups that did not meet the normal distribution, and Kruskal-Wallis test was used for three groups. Tukey test was used for data with normal distribution in multiple comparisons between groups and Dunn's test was used for analysis of the data that did not meet the normal distribution. The summary values of normally distributed continuous variables were given as mean \pm standard deviation and continuous variables that were not normally distributed were given as median (Q1-Q3). The relationship between categorical variables was investigated by chi square analysis. Probability values of $\mathrm{p}<0.05$ were considered statistically significant.

\section{Results}

Patients included in our study were divided into 3 groups as follows: group I - newly diagnosed type $1 \mathrm{DM}$, group II - formerly diagnosed type $1 \mathrm{DM}$, and group III - healthy volunteers. The gender distribution (male/female) in groups I, II and III was $33 / 17,28 / 22$ and $27 / 33$, respectively. The mean age of the patients was 27 years (22-34.2) in group I, 29 years (22.7-35) in group II, and 29 years (24-34.7) years in group III (Tab. 1). Biochemical parameters of the patients are given in Table 2 .

Twenty-five of 100 patients ( $25 \%$ ) with type 1 diabetes included in the study had ATD, $28 \%$ of patients were APA-positive, 10 $\%$ were anti-tissue transglutaminase IgA positive, and $2 \%$ patients

Tab. 2. Biochemical parameters of the study population.

\begin{tabular}{lc}
\hline & mean \pm SS $(\mathrm{n}=160)$ \\
\hline 25(OH)D $(25-80 \mathrm{ng} / \mathrm{ml})$ & $17.9 \pm 11.6$ \\
Calcium $(8.6-10.2 \mathrm{mg} / \mathrm{dl})$ & $9.48 \pm 0.5$ \\
Phosphorus $(2.7-4.5 \mathrm{mg} / \mathrm{dl})$ & $3.53 \pm 0.68$ \\
ALP $(40-130 \mathrm{U} / \mathrm{L})$ & $120 \pm 71$ \\
PTH $(15-65 \mathrm{pg} / \mathrm{ml})$ & $38.6 \pm 15.8$ \\
TSH $(0.27-4.2 \mathrm{uIU} / \mathrm{ml})$ & $2.4 \pm 1.93$ \\
fT4 $(0.93-1.7 \mathrm{ng} / \mathrm{dl})$ & $1.28 \pm 0.68$ \\
Vitamin B12 $(197-771 \mathrm{pg} / \mathrm{ml})$ & $356 \pm 143.9$ \\
Serum kortisol $(6.2-19.4 \mathrm{ug} / \mathrm{dl})$ & $17.2 \pm 5.7$ \\
\hline
\end{tabular}

25(OH)D - 25 hydroxyvitamin D, ALP - alkaline phosphatase, $\mathrm{PTH}$ - parathormone, $\mathrm{TSH}$ - thyroid-stimulating hormone 
Tab. 3. Numerical values of autoimmune disease markers in all patients and in control group included in the study

\begin{tabular}{|c|c|c|c|c|}
\hline Type 1 DM & & $\mathrm{n}=100(\%)$ & Control $\mathrm{n}=60(\%)$ & $\mathrm{p}$ \\
\hline \multirow{2}{*}{ Autoimmune thyroid disease } & positive & $25(25 \%)$ & $6(10 \%)$ & $0.020 *$ \\
\hline & negative & $75(75 \%)$ & $54(90 \%)$ & \\
\hline \multirow{2}{*}{ Anti-tissue transglutaminase IgA } & positive & $10(10 \%)$ & $1(1.7 \%)$ & $0.044^{*}$ \\
\hline & negative & $90(90 \%)$ & & \\
\hline \multirow{2}{*}{ Anti-parietal antibody } & positive & $28(28 \%)$ & $10(16.7 \%)$ & 0.103 \\
\hline & negative & $72(72 \%)$ & $50(83.3 \%)$ & \\
\hline \multirow{2}{*}{ Vitiligo } & positive & $2(2 \%)$ & 0 & 0.270 \\
\hline & negative & $98(98 \%)$ & $60(100 \%)$ & \\
\hline
\end{tabular}

Tab. 4. Comparison of median value of vitamin D according to autoimmune status in all cases.

\begin{tabular}{|c|c|c|c|}
\hline & \multicolumn{2}{|c|}{$25(\mathrm{OH}) \mathrm{D}(\mathrm{ng} / \mathrm{ml})$} & $\mathrm{p}$ \\
\hline Autoimmune thyroid disease & $\begin{array}{c}\text { positive }(n=31) \\
\text { negative }(n=129)\end{array}$ & $\begin{array}{c}11.4(7.2-19) \\
15.3(10.4-23.9)\end{array}$ & $0.017 *$ \\
\hline Anti-parietal antibody & $\begin{array}{c}\text { positive }(n=38) \\
\text { negative }(n=122)\end{array}$ & $\begin{array}{c}12.2(8.7-19.3) \\
15.1(10-23.9)\end{array}$ & 0.133 \\
\hline Anti-tissue transglutaminase IgA & $\begin{array}{c}\text { positive }(n=11) \\
\text { negative }(n=149)\end{array}$ & $\begin{array}{c}15(11-25) \\
14(9.5-22.5)\end{array}$ & 0.377 \\
\hline
\end{tabular}

Tab. 5. Comparison of autoimmune disorders between groups.

\begin{tabular}{|c|c|c|c|c|c|}
\hline & & Group I n=50 (\%) & Group II $n=50(\%)$ & Group III n=60 (\%) & $\mathrm{p}$ \\
\hline \multirow{2}{*}{ APA } & positive & $15(30 \%)$ & $13(26 \%)$ & $10(16.7 \%)$ & 0.237 \\
\hline & negative & $35(70 \%)$ & $37(74 \%)$ & $50(83.3 \%)$ & \\
\hline \multirow{2}{*}{ Anti-tissue TG-IgA } & positive & $5(10 \%)$ & $5(10 \%)$ & $1(1.7 \%)$ & 0.131 \\
\hline & negative & $45(90 \%)$ & $45(90 \%)$ & $59(98.3 \%)$ & \\
\hline \multirow{2}{*}{ ATD } & positive & $12(24 \%)$ & $13(26 \%)$ & $6(10 \%)$ & 0.065 \\
\hline & negative & $38(76 \%)$ & $37(74 \%)$ & $54(90 \%)$ & \\
\hline Vitiligo & $\begin{array}{l}\text { positive } \\
\text { negative }\end{array}$ & $\begin{array}{c}1(2 \%) \\
49(98 \%)\end{array}$ & $\begin{array}{c}1(2 \%) \\
49(98 \%)\end{array}$ & $\begin{array}{c}0(0 \%) \\
60(100 \%)\end{array}$ & 0.545 \\
\hline
\end{tabular}

APA - anti-parietal antibody, ATD - autoimmune thyroid disease

had vitiligo skin findings. In control group of healthy volunteers, ATD was found in 6 subjects (10\%), APA positivity in $10(16.7 \%)$, and anti-tissue transglutaminase IgA positivity in 1 (1.7\%). Vitiligo skin signs were found in no healthy volunteers (Tab. 3).

The mean level of 25(OH)D was $14.6 \mathrm{ng} / \mathrm{dL}(8.7-19.7)$ in group I, $12.1 \mathrm{ng} / \mathrm{dL}$ (8.1-20.2) in group II, and 16.1 ng/dL (11.9$28.9)$ in group III. There was a statistically significant difference between groups I and III and between groups II and III at $25(\mathrm{OH})$ D levels $(\mathrm{p}<0.05)$. In all diabetic patients (Groups I + II), 25(OH) $\mathrm{D}$ levels were lower than in group III.

In all cases included in the study, $25(\mathrm{OH}) \mathrm{D}$ levels were compared with autoimmune disorders (Tab. 4). The median 25(OH)D level was $11.4 \mathrm{ng} / \mathrm{ml}(7.2-19 \mathrm{ng} / \mathrm{ml})$ in patients with ATD, while the median 25(OH)D was $15.3 \mathrm{ng} / \mathrm{ml}(10.4-23.9 \mathrm{ng} / \mathrm{ml})$ and a statistically significant difference was detected $(\mathrm{p}<0.05)$. The level of $25(\mathrm{OH}) \mathrm{D}$ was lower than in the negative ones in APA-positive cases and there was no statistically significant difference $(p>0.05)$. No statistically significant difference was found between anti-tissue transglutaminase IgA antibody positivity and $25(\mathrm{OH}) \mathrm{D}(\mathrm{p}>0.05)$. There was no statistically significant difference between groups in terms of ATD, APA, anti-tissue transglutaminase IgA, and vitiligo positivity and negativity ( $\mathrm{p}>0.05$ ) (Tab. 5).

ATD was observed in 25 subjects ( $25 \%$ ) from type 1 diabetic patients included in our study and in $6(10 \%)$ from the control group while there was a statistically significant difference ob- served $(\mathrm{p}<0.05)$. Anti-tissue transglutaminase IgA positivity was observed in $10(10 \%)$ of patients and in 1 volunteer from the control group $(1.7 \%)$. There was a statistically significant difference between the latter two groups $(\mathrm{p}<0.05)$. There was no statistically significant difference between the groups when APA and vitiligo positivity and negativity were taken into account ( $\mathrm{p}$ $>0.05$ ) (Tab. 6).

When vitamin D deficits were compared between the groups, vitamin D deficiency was detected in 44 of 50 patients ( $88 \%$ ) in the newly diagnosed type $1 \mathrm{DM}$ group, in 42 of 50 patients ( 84 $\%$ ) from the formerly diagnosed type $1 \mathrm{DM}$ group, and in 40 of 60 patients $(66.7 \%)$ from control group. According to these results, there was a statistically significant difference between the control group with newly diagnosed type $1 \mathrm{DM}$ group and the formerly diagnosed type $1 \mathrm{DM}$ group with control group in terms of $25(\mathrm{OH})$ D deficiency $(\mathrm{p}<0.05)$ (Tab. 7).

When APA positivity was assessed in all cases included in the study, 11 of 160 cases $(6.8 \%)$ were found to to be both ATDand APA-positive. These cases constituted $35 \%$ of 31 cases with ATD and $29 \%$ of 38 APA-positive cases. There was no statistically significant relationship between ATD and APA positivity and negativity in any of cases $(\mathrm{p}>0.05)$.

Among all cases included in the study, 25(OH)D levels were compared between those with both ATD and APA positivity and those who were negative. The median $25(\mathrm{OH}) \mathrm{D}$ was $11 \mathrm{ng} / \mathrm{ml}$ 
Tab. 6. Comparison of all patients and control group in terms of autoimmune disorders.

\begin{tabular}{llccc}
\hline & & $\begin{array}{c}\text { Type 1 DM } \\
\mathrm{n}=100(\%)\end{array}$ & $\begin{array}{c}\text { Control } \\
\mathrm{n}=60(\%)\end{array}$ & $\mathrm{p}$ \\
\hline \multirow{2}{*}{ ATD } & positive & $25(25 \%)$ & $6(10 \%)$ & $0.020^{*}$ \\
& negative & $75(75 \%)$ & $54(90 \%)$ & \\
\hline \multirow{2}{*}{ Anti-tissue TG-IgA } & positive & $10(10 \%)$ & $1(1.7 \%)$ & $0.044 *$ \\
& negative & $90(90 \%)$ & $59(98.3 \%)$ & \\
\hline \multirow{2}{*}{ APA } & positive & $28(28 \%)$ & $10(16.7 \%)$ & 0.103 \\
& negative & $72(72 \%)$ & $50(83.3 \%)$ & \\
\hline \multirow{2}{*}{ Vitiligo } & positive & $2(2 \%)$ & 0 & 0.270 \\
& negative & $98(98 \%)$ & $60(100 \%)$ & \\
\hline
\end{tabular}

ATD - autoimmune thyroid disease, APA - anti-parietal antibody

Tab. 7. Comparison of patients with and without vitamin D deficiency according to the groups.

\begin{tabular}{cccccc}
\hline & Group I & Group II & Group III & p \\
& $n(\%)$ & $n(\%)$ & $n(\%)$ & \\
\hline $25(\mathrm{OH}) \mathrm{D}(<25 \mathrm{ng} / \mathrm{ml}$ & $44(88 \%)$ & $42(84 \%)$ & $40(66.7 \%)$ & $\mathrm{I}-\mathrm{III} \rightarrow^{*}$ \\
& $\geq 25 \mathrm{ng} / \mathrm{ml}$ & $6(12 \%)$ & $8(16 \%)$ & $20(33.3 \%)$ & $\mathrm{II}-\mathrm{III} \rightarrow *$ \\
\hline
\end{tabular}

$(5-11.9 \mathrm{ng} / \mathrm{ml})$ in subjects positive for both conditions, while in patients negative for both conditions, the median $25(\mathrm{OH}) \mathrm{D}$ was $15.3 \mathrm{ng} / \mathrm{ml}(5-25 \mathrm{ng} / \mathrm{ml})$. There was a statistically significant difference in the median value of $25(\mathrm{OH}) \mathrm{D}$ between patients with ATD and concurrent APA positivity and those being negative for both conditions $(p<0.05)$. No statistically significant difference was found between the presence of ATD, anti-tissue transglutaminase positivity and vitamin D levels in the newly diagnosed type 1 diabetes group. However, it was observed that APA positivity made a statistically significant difference in D vitamin levels in the same group.

When ATD and APA were compared in all subjects included in the study, 10 of 100 patients ( $10 \%$ ) had both diabetes and were APA-positive. These patients constituted $40 \%$ of 25 patients with ATD and $35.7 \%$ of 28 APA-positive patients. There was no statistically significant relationship between ATD and APA positivity and negativity in any patients $(\mathrm{p}>0.05)$.

The levels of $25(\mathrm{OH}) \mathrm{D}$ of all patients included in the study were compared with those with concurrent diabetes and APA positivity and those negative for both latter conditions. The median level of vitamin D in patients with ATD and APA positivity was $9.8 \mathrm{ng} / \mathrm{ml}(5-13.9 \mathrm{ng} / \mathrm{ml})$ the median level of vitamin D in those who did not have ATD and APA positivity was $14.3 \mathrm{ng} / \mathrm{ml}$ (9.5-21 ng/ml). Patients who had both ATD and were concurrently APA-positive were found to have a lower median value of $25(\mathrm{OH})$ $\mathrm{D}$ than patients who did not have ATD and were not APA-positive, but no statistically significant difference was found $(\mathrm{p}>0.05)$.

\section{Discussion}

Type $1 \mathrm{DM}$ is a chronic metabolic disease characterized by absolute insulin insufficiency resulting from autoimmune destruction of beta cells mediated by T-cell. The origin of the disease in some cases may be idiopathic. The condition leads to hyperglycemia. Studies have suggested that vitamin D plays a role in the devel- opment of type $1 \mathrm{DM}$ because of the close relationship between ultraviolet $B$ radiation, the primary source of vitamin D synthesis, and incidence of type $1 \operatorname{DM}(5)$. Studies have shown that in the early years of life, the risk of developing type $1 \mathrm{DM}$ can be reduced with vitamin D supplementation $(6,7,8)$. In a study conducted by Gabbay et al, it has been shown that daily supplementation of 2000 IU vitamin D for 18 months reduced the reduction of residual beta-cell function in newly diagnosed type I diabetic patients (9).

Vitamin D deficiency is common in patients with type 1 diabetes. In a study conducted in North India, the incidence of vitamin $\mathrm{D}$ deficiency was reported in $58 \%$ of children with type 1 diabetes included in the study group and $32 \%$ of those in healthy control group (10). In a study conducted in Sweden by Littorin et al, 459 newly diagnosed diabetic patients aged between 15 and 34 years were found to have lower levels of vitamin D than 208 healthy control subjects similar as to age and area of residence, and it was suggested that vitamin D may play a role in the development of type 1 diabetes (11). In the meta-analyzes of Shen et al, involving 2,003 subjects with type $1 \mathrm{DM}$ and 1,882 control patients, 25 $(\mathrm{OH}) \mathrm{D}$ vitamin levels in patients with type 1 diabetes were found to be lower than in the control group (12).

Of the total 100 patients and 60 control subjects included in our study, 126 (78\%) had vitamin D deficiency. Vitamin D deficiency was detected in 44 (88\%) of 50 patients with a newly diagnosed type 1 diabetes, 42 (84\%) of 50 patients with formerly diagnosed type 1 diabetes and $40(66.7 \%)$ of 60 subjects from the control group. It was found that the prevalence of vitamin D deficiency was significantly increased in both newly and formerly diagnosed type $1 \mathrm{DM}$ groups compared to the control. While the median D vitamin level was lowest in the formerly diagnosed type $1 \mathrm{DM}$ group, no statistically significant difference was found when compared with the newly diagnosed type $1 \mathrm{DM}$ group.

Autoimmune diabetes and autoimmune thyroid disease are commonly associated with some susceptible genotypes in HLA that are involved in immune regulation and other genes $(13,14)$ while patients with type 1 diabetes are at increased risk of autoimmune thyroiditis, Graves and hypothyroidism. A proportion of 15-30 \% of patients with type 1 diabetes have autoimmune thyroid disease. Anti-TPO positivity is also seen in $2-10 \%$ of healthy adults ( 15 , 16). In a study published by Sakly et al in 2011 , the presence of autoimmune thyroid disease was found to be $14 \%$ in patients with type 1 diabetes and $2.8 \%$ in healthy control group. In the HUNT2 study conducted by Fleiner et al in 2015, anti-TPO positivity was found in $27.5 \%$ of type I diabetic female patients and in $16.9 \%$ of type I diabetic male patients whereas anti-TPO positivity was found in $19.4 \%$ of healthy female patients and $5 \%$ of healthy male patients. According to these studies, there was a significant difference in the presence of autoimmune thyroid disease between the healthy control group and diabetic group $(17,18)$.

In our study, the incidence of ATD was $25 \%$ of all patients, $24 \%$ of the newly diagnosed type 1 DM group, $26 \%$ of the formerly diagnosed type $1 \mathrm{DM}$ group and $10 \%$ of the healthy control group. There was no significant difference between the groups, but when the diabetics were considered as a whole, it was determined that the frequency of ATD was statistically significantly 
higher than that in the control group. In accord with the study of HUNT2, our study found that ATD was present in $33 \%$ of female diabetic patients, $19.6 \%$ of male diabetic patients, $18 \%$ of female healthy controls and $3 \%$ of male healthy controls. Our study also revealed subclinical hypothyroidism in $44 \%$ of all patients with type 1 diabetes and ATD, whereas thyroiditis was detected in $56 \%$ of these patients by means of thyroid ultrasound.

Especially in recent years, studies have shown that vitamin $\mathrm{D}$ deficiency is also increased in autoimmune thyroid diseases. In the study performed by Bozkurt and his colleagues, vitamin D deficiency was found in $48.3 \%$ of 180 newly diagnosed autoimmune thyroid patients, $35 \%$ of 180 formerly diagnosed autoimmune thyroid patients and in $20.5 \%$ of 180 healthy control patients while the lowest average vitamin D level was found in the newly diagnosed autoimmune thyroid patients (19). While there are many studies in literature on autoimmune thyroid disease and vitamin $\mathrm{D}$ association, there is no study of the association of type $1 \mathrm{DM}$ with autoimmune thyroid disease vitamin $\mathrm{D}$.

In agreement with other studies, $25(\mathrm{OH}) \mathrm{D}$ levels were found to be statistically significantly lower in patients with ATD than in patients without ATD, suggesting that vitamin D deficiency may be a risk factor for ATD. In the newly diagnosed type 1 DM group, $25(\mathrm{OH}) \mathrm{D}$ levels were found to be lower in patients with ATD than in those without ATD, but no statistically significant difference was observed. There was no significant correlation between serum $\mathrm{TSH}$, free T4 levels and 25(OH) D in the same group.

There is an increased prevalence of autoimmunity against non-pancreatic endocrine tissues as well as against non-endocrine gastric and enteral mucosa in type $1 \mathrm{DM}$. APA positivity is $4-5$ times more common in patients with type 1 diabetes than in the non-diabetic population. In a study conducted by Christophe et al, APA positivity was detected in $20.9 \%$ of 497 type 1 diabetic patients while no gender difference was observed. Anti-TPO was more prevalent than in non-APA-positive group (20). Erten et al found APA positivity in $8.2 \%$ of 73 newly diagnosed type 1 diabetic patients and in $3.6 \%$ of subjects in healthy group. In the same study, there was a positive correlation between the presence of APA and anti-TPO in newly diagnosed type 1 diabetes (21).

Anti-parietal cell antibody positivity indicating a background of pernicious anemia was detected in $28 \%$ of patients with type 1 diabetes who had been included in the study. Out of the patients positive for anti-parietal cell antibody, $46 \%$ had B12 deficiency and $14 \%$ had anemia. In our study, $25(\mathrm{OH}) \mathrm{D}$ was found to be lower in APA-positive patients than in those APA-negative. Nevertheless, the difference was not statistically significant. While there was no difference in B12 vitamin levels between the groups, no significant correlation was found between B12 vitamin and $25(\mathrm{OH})$ $\mathrm{D}$ in the patient groups.

Out of the 160 cases included in our study and 100 diabetic patients, both ATD and APA positivity were present in $11(6.8 \%)$ and $10(10 \%)$, respectively. APA positivity was also detected in $35 \%$ of cases with ATD while ATD positivity was found at the same time in $29 \%$ of cases with APA positivity. APA positivity was found in $40 \%$ of patients with ATD and ATD positivity was found in $37.5 \%$ of patients with APA positivity in all T1DM patients included in the study. Considering the prevalence of pernicious anemia and ATD in the general population, the above values did not show a statistically significant relationship although both autoimmune disorders were thought to occur more frequently together.

Out of 160 cases, 25(OH)D levels were statistically significantly lower in patients with both ATD and APA positivity than in those negative for both conditions. Out of 100 diabetic patients included in the study, 25(OH)D levels were lower in patients with both autoimmune disorders than in those without. Nevertheless, no statistically significant relationship was found. These findings may suggest that as the severity of vitamin D deficiency increases, more than one autoimmune disorder co-occurs, or that autoimmune diseases contribute to the development of other autoimmune disorders over time. However, since there is no trial in literature regarding APA, pernicious anemia, atrophic gastritis in association with vitamin $\mathrm{D}$, it is concluded that new studies including more patients are needed.

The incidence of clinical or subclinical forms of celiac disease has increased in type 1 diabetes. Celiac autoimmunity can be detected by investigating the anti-IgA autoantibody against tissue transglutaminase. The prevalence of celiac disease in type 1 diabetes is $8 \%$, while being $0.5 \%$ in the healthy population ( 22 , 23). The incidence of vitamin D deficiency has increased in type 1 DM and ATD, while celiac disease is commonly concomitant with latter diseases. In one study, there was no significant difference in 25(OH)D levels between type 1 diabetic patients with anti-tissue IgA positivity and those without anti-tissue IgA positivity (24).

In our study, IgA positivity was found in $10 \%$ of 100 patients included in the study and $1.7 \%$ of 60 control patients. A statistically significant difference was observed between the groups. In all the patients included in the study, 25(OH)D did not show a statistically significant difference in anti-tissue IgA-positive patients compared to those without anti-tissue TG-IgA positivity. In the newly diagnosed type $1 \mathrm{DM}$ group, $25(\mathrm{OH}) \mathrm{D}$ vitamin levels were similar among patients with and without anti-tissue TG-IgA.

The prevalence of AD in type $1 \mathrm{DM}$ was found to be $0.5 \%$. The incidence of adrenocortical autoantibodies in Type $1 \mathrm{DM}$ ranges from 0.5 to $3 \%$, and in healthy adults it ranges from 0 to $0.6 \%$ (25). Prazny et al found anti-adrenal antibody positivity in 2 of 51 type 1 diabetic patients while none of them had Addison disease (26). Whether or not there is a difference in $25(\mathrm{OH}) \mathrm{D}$ concentrations between those with and without autoimmune $\mathrm{AD}$ is unknown and is still under investigation (27). When clinical findings and basal cortisol levels were considered, $\mathrm{AD}$ was found in none of patients included in our study. Serum cortisol level was higher in the newly diagnosed type $1 \mathrm{DM}$ group, and it was higher in type 1 DM group than in control group, nevertheless with no statistically significant difference between the groups. This is thought to be due to the cortisol hormone functioning as a counter-regulator. There was a very weak negative correlation between serum $25(\mathrm{OH})$ $\mathrm{D}$ and serum cortisol in the newly diagnosed type $1 \mathrm{DM}$ group, but no significant correlation was found between 25(OH)D and cortisol levels in the other groups.

Vitiligo is found in $0.5 \%$ of the healthy population, while in type 1 diabetes group, this rate increases up to $2-8 \%(25)$. The 
incidence of other autoimmune diseases has increased in patients with vitiligo, while ATD and autoimmune gastritis are associated with vitiligo in $30 \%$ and $15 \%$ of patients, respectively $(28,29$, $30)$. There were only two patients with vitiligo in our study while one of them was in the newly diagnosed type 1 DM group and the other in the formerly diagnosed type 1 DM group. Simultaneous ATD and anti-tissue TG IgA positivity were detected in the subject from the newly diagnosed type $1 \mathrm{DM}$ group. In the other case from the formerly diagnosed type 1 DM group, there was accompanying ATD. Patients with vitiligo were not included in the statistical analysis due to an inadequate number of patients.

In our study, in newly diagnosed type 1 diabetic patients, the prevalence of anti-parietal cell antibody positivity indicating pernicious anemia, ATD, anti-tissue transglutaminase TG-IgA antibody indicating celiac disease and vitiligo were $30 \%, 24 \%, 10$ $\%$ and $2 \%$, respectively. The levels of $25(\mathrm{OH}) \mathrm{D}$ in APA-positive newly diagnosed type 1 diabetics were significantly lower than in those APA-negative.

As a result, the prevalence of vitamin D deficiency in newly diagnosed type 1 diabetic was $88 \%, 84 \%$ in formerly diagnosed type 1 diabetic, and $66.7 \%$ in healthy volunteers. In this study, the vitamin D level was lower in (i) type 1 diabetic patients when compared with healthy subjects, (ii) all cases included in the study with ATD when compared with those without ATD, (iii) all APA-positive type 1 diabetic patients with ATD when compared with none (iv) APA-positive newly diagnosed type 1 diabetics when compared with those APA-negative.

\section{References}

1. Rosen CJ, Adams JS, Bikle DD et al. The Nonskeletal Effects of Vitamin D: An Endocrine Society Scientific Statement. Endocrine Rev 2012; 33 (3): 456-492.

2. Larsson HE, Ivarsson S, Lernmark A. A Case-Based Guide To Clinical Endocrinology Chapter 37 Type 1 Diabetes and Comorbidity of Addison's Disease. Humana Press Totowa NJ 200814 (4): 337.

3. Takiishi T et al. Vitamin D and Diabetes. Endocrinol Metab Clin North Amer 2010; 39 (2): 419-447.

4. Feng $\mathbf{R}$ et al. Lower serum $25(\mathrm{OH}) \mathrm{D}$ concentrations in type 1 diabetes: A meta-analysis. Diabet Res Clin Pract 2015; 108 (3): 71-75.

5. Mohr SB et al. The association between ultraviolet B irradiance, vitamin $\mathrm{D}$ status and incidence rates of type 1 diabetes in 51 regions worldwide.

6. Hyppönen $\mathrm{E}$ et al. Intake of vitamin $\mathrm{D}$ and risk of type 1 diabetes: a birthcohort study. Lancet 2001; 358: 1500-1503.

7. The EURODIAB Substudy 2 Study Group. Vitamin D supplement in early childhood and risk for type I (insulin-dependent) diabetes mellitus. Diabetologia 1999; 42: 51-54.

8. Dong JY et al. Vitamin D Intake and Risk of Type 1 Diabetes: A MetaAnalysis of Observational Studies. Nutrients 2013; 5 (9): 3551-3562.

9. Gabbay MA et al. Effect of cholecalciferol as adjunctive therapy with insulin on protective immunologic profile and decline of residual beta-cell function in new-onset type 1 diabetes mellitus. Arch Pediatr Adolesc Med 2012; 166 (7): 601-607.

10. Borkar VV, Devidayal VS, Bhalla AK. Low levels of vitamin D in North Indian children with newly diagnosed type 1 diabetes. Pediatrc Diabet 2010; 11 (5): 345-350.
11. Littorin B et al. Lower levels of plasma 25-hydroxyvitamin D among young adults at diagnosis of autoimmune type 1 diabetes compared with control subjects: results from the nationwide Diabetes Incidence Study in Sweden (DISS). Diabetologia 2006; 49: 2847-2852.

12. Shen L, Zhuang QS, and Ji HF. Assessment of vitamin D levels in type 1 and type 2 diabetes patients: Results from meta-analysis. Mol Nutr Food Res 2016.

13. Dittmar M, Kahaly GJ. Genetics of the autoimmune polyglandular syndrome type 3 variant. Thyroid 2010; 20: 737-743.

14. Tomer $Y$ et al. Genome wide identification of new genes and pathways in patients with both autoimmune thyroiditis and type 1 diabetes. J Autoimmun 2015; 60: 32-39.

15. Shun CB et al. Thyroid autoimmunity in Type 1 diabetes: systematic review and meta-analysis. Diabet Med 2014; 31: 126-135.

16. Van den Driessche A et al. Type 1 diabetes and autoimmune polyglandular syndrome: a clinical review. Neth J Med 2009; 67: 376-387.

17. Fleiner HF et al. Prevalence of Thyroid Dysfunction in Autoimmune and Type 2 Diabetes: The Population-Based HUNT Study in Norway. J Clin Endocrinol Metab 2016; 101 (2): 669-677.

18. Sakly W et al. Thyroid-related autoantibodies in Tunisian patients with type 1 diabetes. Endocr Res 2012; 37 (2): 59-66.

19. Bozkurt NC et al. The association between severity of vitamin D deficiency and Hashimoto's thyroiditis. Endocr Pract 2013; 19 (3): 479-484.

20. De Block CE et al. High prevalence of manifestations of gastric autoimmunity in parietal cell antibody-positive type 1 (insulin-dependent) diabetic patients. The Belgian Diabetes Registry. J Clin Endocrinol Metab 1999; 84 (11): 4062-4067.

21. Erten $\mathbf{G}$ et al. Organ specific autoantibodies in preclinical and early clinical type 1 diabetes in Turkey. Ups J Med Sci 2007; 112 (2): 231-243.

22. Gillett PM et al. High prevalence of celiac disease in patients with type 1 diabetes detected by antibodies to endomysium and tissue transglutaminase. Can J Gastroenterol 2001; 15 (5): 297-301.

23. Volta U, Fabbri A, Parisi C et al. Old and new serological tests for celiac disease screening. Expert Rev Gastroenterol Hepatol 2010; 4: 31-35.

24. Simmons KM et al. Reduced Bone Mineral Density Is Associated with Celiac Disease Autoimmunity in Children with Type 1 Diabetes. J Pediatr 2016; 169: 44-48.

25. Van den Driessche A et al. Type 1 diabetes and autoimmune polyglandular syndrome: a clinical review. Neth J Med 2009; 67 (11): 376-387.

26. Prazny $M$ et al. Screening for associated autoimmunity in type 1 diabetes mellitus with respect to diabetes control. Physiol Res 2005; 54 (1): 41-48.

27. Muscogiuri G et al. Mechanisms in Endocrinology: Vitamin D as a potential contributor in endocrine health and disease. Eur J Endocrinol 2014; 171: $101-110$.

28. Kakourou $\mathrm{T}$ et al. Increased prevalence of chronic autoimmune (Hashimoto's) thyroiditis in children and adolescents with vitiligo. J Am Acad Dermatol 2005; 53: 220-223.

29. Alkhateeb A et al. Epidemiology of vitiligo and associated autoimmune diseases in Caucasian probands and their families. Pigment Cell Res 2003; 16: 208-214.

30. Betterle $\mathrm{C}$ et al. Incidence and significance of organ-specific autoimmune disorders (clinical, latent or only autoantibodies) in patients with vitiligo. Dermatologica 1985; 171: 419-423.

Received April 13, 2018. Accepted May 25, 2018. 